\title{
Introducing Virtuality to Enhance Game-related Physical Artifacts
}

\author{
Mizuki Sakamoto, Todorka Alexandrova, Tatsuo Nakajima \\ Department of Computer Science and Engineering \\ Waseda University \\ \{mizuki,toty,tatsuo\}@dcl.cs.waseda.ac.jp
}

\begin{abstract}
Various smart artifacts that embed computers and sensors have been developed. Some of these artifacts include extra hardware components to enhance their functionalities, but the components are always real, not virtual. Ubiquitous computing technologies for introducing virtuality offer the possibility of adding virtual components to physical arfifacts, but this possibility has not been sufficiently exploited in previous research. In this paper, we present the Augmented Trading Card Game (Augmented TCG) as a case study for enhancing physical artifacts by integrating virtuality. Augmented TCG montors the opponent's movement using MS Kinect and maps them onto a virtual character representing the opponent rather than directly displaying the real opponent's actons An image of the opponent's card is also projected onto the table in front of the player. Thus, the player feels as if he/she is playing face-to-face with the opponent. Moreover virtuality allows more information to be displayed during game play. We conducted seyenal experiments to examine how players feel while playing a game,, and we present 4 ugmented TCG experiences as a case study in integrating virtuality into physical artifacts.
\end{abstract}

Keywords: Augmenteprealty; Ambient intelligence; Digitally enhanced artifacts; Trading card game; Physical tangibility; Incorporating Virtuality; Gamification

\section{Introduction}

The physical artifacts that surround us are continuously becoming more intelligent $[6,11$, $14,17,21]$ due to ubiqutous computing technologies and to the embedding of computers and sensors into these arifacts. These artifacts use contextual information from our surroundings, and integrating additional actuators to the artifacts creates advanced functionality. Integrating virtual objects into these physical artifacts offers new opportunities for enhanced functionality. For example. AwareMirror [12] presents information on a mirror in a lavatory that can help us to make better choices in our daily lives. A virtual object is defined as something that does not really exist but that has a real effect on our daily life as if it did exist. Baudrillard described our consumption behavior as the consumption of the symbols associated with things and not of the things themselves [1]. Our virtual consumption will increase rapidly as symbolization is accelerated by the embedding of computers in our lives. The embedding of computers in the real world allows physical artifacts to be easily enhanced using virtual objects; however, these possibilities have not been widely explored.

The impact of introducing virtuality in our daily lives has not been thoroughly discussed; although, digital books have become common, and virtual worlds such as that in The Elder 
Scrolls V: Skyrim ${ }^{1}$ are almost indistinguishable from the real world. It is important to examine the effects of replace real things with virtual things. In modern games, physically tangible things are replaced by virtual things, and multiple players can easily enjoy playing a game remotely without meeting each other. Although the rules of the game do not change, the style of play is significantly affected by the game's realization method [22].

In this paper, we present the Augmented Trading Card Game (Augmented TCG). Augmented TCG enhances the original TCG by using virtuality to support remote play. Images of the opponent's physical cards are projected onto the table in front of the player. The opponent is represented by a virtual character. The virtual character on the player's card is also shown on a small display, and this character encourages the player.

We conducted a user study in which we observed the participants' behavior during play and interviewed them after the game. The study examined the impact of introducing virtuality into a TCG game played with real cards. In particular, we were interested in the effect of using popular virtual characters from Japanese animation and games to represent opposing players. The findings of this study will be useful for enhancing other physical artifacts with virtuality through ubiquitous computing technologies. Many previous case studies have examined the incorporation of virtuality into physical artifacts [12, 19, 27], but our approach is unique in that we have incorporated popular virtual characters into physical artifacts and have used an Augmented TCG as a case study to examine the impact of this incorporation.

The remaining sections are structured as follows. Section 2 discusses previous studies that are related to our research. Section 3 presents an overview of $y_{a} G i-O h ! T C G$ and its remote play set-up. Section 4 presents an overview of Augmented TCG, and the experimental results are described in Section 5. Section 6 describes how virtulaty can be enhanced by the concept of transmedia storytelling. Finally, Section 7 conclâdes the paper.

\section{Related Work}

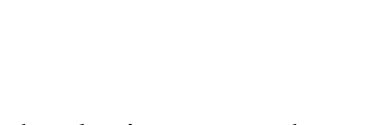

Ubiquitous computing technologies can enhance the TCG gaming experience. Through remote TCG play, computers offer new possibilities to extend the original TCG play. The Eye of Judgment ${ }^{2}$ uses augmented reality technologies to display special effects on the real trading cards. This technology allows for special effects to be shown on physical cards that are used by the players In Augmented TCG, it is easy to add special effects to the card images that are projected onto the table in front of a player. Duel Accelerator ${ }^{3}$ is an online-based $\mathrm{Yu}$-Gi-Oh! $T C G$ where each player chooses his/her avatar and virtual trading cards, which display special effects during the duel, are used. The special effects are an emotional stimulus for the player, and thus the pleasure of the game is increased. However, virtual cards lose the sense of the physical tangibility of the cards, which makes it difficult to motivate players to enjoy collecting cards [22]. Additionally, Skype duel uses Skype to show each player's cards on the opposite player's display; and voice communication between the two players is also possible. Augmented ICG uses 3D virtual characters that are shown to the players, and the characters move according to the opponent's actions.

In the new TCG CyberOne $e^{4}$ each paper card has a sequence number. When the number is entered into the system, the corresponding virtual card appears in the online TCG. The player can enjoy the tangibility of the cards as well as additional special effects, which is an advantage of the virtual cards. Once the full set of cards in the player's deck is entered into

\footnotetext{
${ }^{1} \mathrm{http}: / /$ www.elderscrolls.com

${ }^{2} \mathrm{http} / / /$ en.wikipedia.org/wiki/The_Eye_of_Judgment

${ }^{3} \mathrm{http}: / / \mathrm{www}$.yugioh-online.net/v3/newvisitors/

${ }^{4}$ http://www.c-1cyberone.com/official_top/official_top.html
} 
the system, the duel occurs automatically without any further action of the player, and the final result of the duel is returned to the player, showing the strength of his/her deck selection. The enjoyment of constructing an original deck still remains for the player. Another advantage is that the two players do not need to be playing at the same time because they only need to construct their decks.

Augmented reality techniques can be used to enhance existing games. For example, [27] describes several augmented reality games that are enhanced versions of traditional physical games. Specifically, Augmented Go [10] demonstrates a promising approach to maintaining the advantages of the physicality of the board game while also adding virtuality. Additionally, in Smart Playing Cards [7], each card contains an RFID tag, which allows the system to keep track of who is using particular cards.

Some pervasive games also incorporate both virtual and tangible objects. For example, in Pac-Man and Ghosts, human players in the real world experience a computer graphícsgenerated fantasy-reality using wearable computers [15]. The basic concepts from pervasive games can also be useful in realizing our goal. As shown in [3], the use of tangible rather than virtual objects in gaming helps to improve human performance in the real world. Our approach can also be used for enhancing a traditional pervasjve game usíng transmedia storytelling. A fictional story can be told across multiple media platforms, which are integrated in our daily lives.

In [22], we discussed how a virtual character is effective when the virtual and real worlds are blended. A virtual character that is used in both an mation and game stories can seamlessly blend real life with the fictional worly of the animation and game stories.

\section{Yu-Gi-Oh! Trading Card Gameand Kemote Play}

The Yu-Gi-Oh! TCG5 is a trading card game based on the Duel Monsters game that is portrayed in the popular $Y u$-Gi-Qh! comic. Y $u-G i O h$ ! cards are categorized into three types: Monster, Spell and Trap cards. A Yu-Gi-Oh! TCG player structures his/her own original deck by selecting his/her favorile ards from the several thousands of $\mathrm{Yu}-\mathrm{Gi}$-Oh! cards that are currently available. This leads to each user having his/her own unique and original deck that reflects his/her own personality and taste.

$\mathrm{Yu}$-Gi-Oh! TCQ is a turn-based game, which is played in a one-to-one or two-to-two manner, as shown in Figure 1. The battle with the Yu-Gi-Oh! Cards is called a duel. Each player starts the game with a certain number of points called life points and performs the duel by summoning his/he monsters and using them, or spells and traps, against the opponent. Depending on the actions taken and their outcomes during the duel, each player's life points decrease or increase. If the life points of one player become zero or he/she cannot draw cards from his/her own deck, then that player loses the duel and the game ends.

$\mathrm{Yu}-\mathrm{Gi}$-Oh TCG involves various sources of enjoyment in addition to playing the game, such as completing card collections, structuring decks, communicating with opponents, trading battling, and establishing different links to $\mathrm{Yu}$-Gi-Oh! TV animations and $\mathrm{Yu}$-Gi-Oh! conics. Although the computerization of the game has advanced recently, the traditional version of $\mathrm{Yu}-\mathrm{Gi}-\mathrm{Oh}$ ! TCG that uses paper cards, and does not use a computer, is still very popular among players.

In Japan, as already described, trading card games such as $\mathrm{Yu}-\mathrm{Gi}-\mathrm{Oh}$ ! TCG are very popular among children, but most of players stop playing as they grow older because they no longer have time to meet and play with other people. However, because of the complicated rules and interesting strategies involved in trading card games, these games are still popular

\footnotetext{
${ }^{5}$ http://www.yugioh-card.com/en/
} 
among adults. Thus, playing trading card games remotely has become typical in our busy modern society. Remote play is also becoming more popular for many other games, such as Go, Chess, Poker and Mah-jong. In this section, we describe some problems and limitations related to existing styles of remote trading card game play.

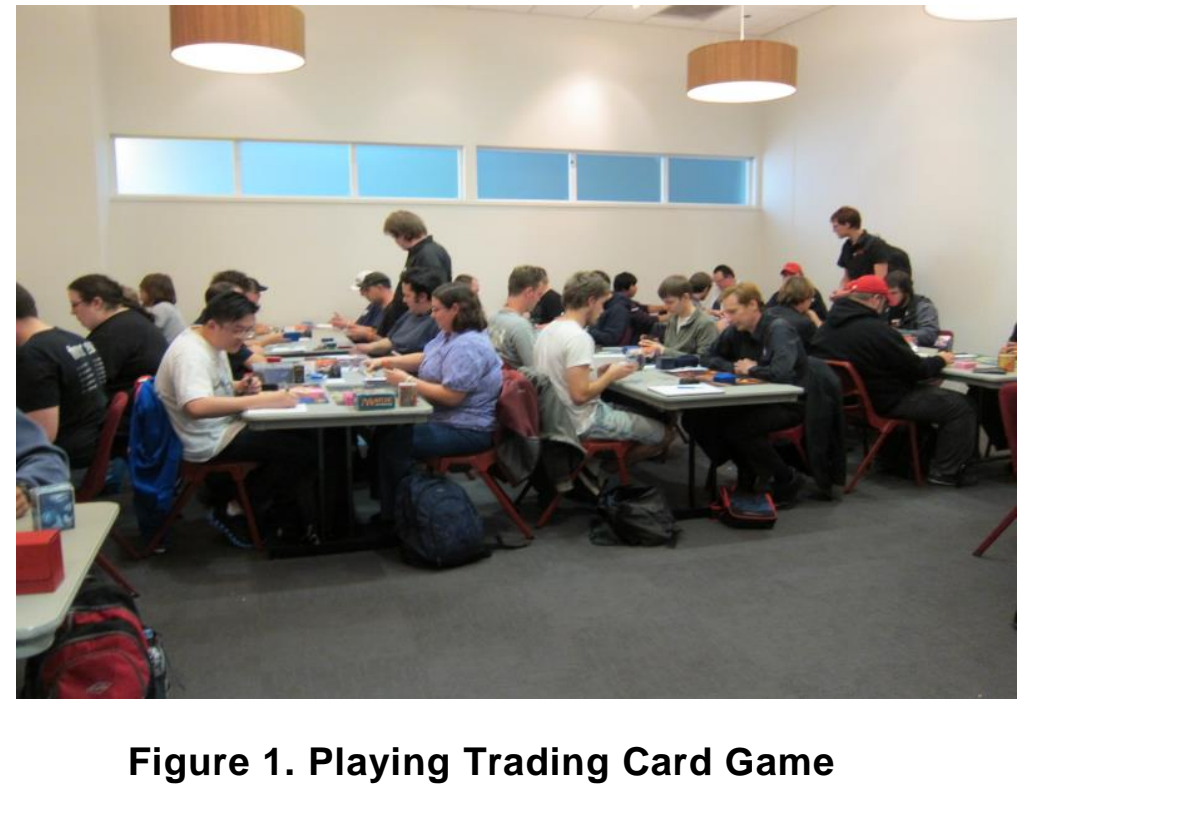

There are already several systems that support remote trading card game play. $\mathrm{Yu}-\mathrm{Gi}-\mathrm{Oh}$ ! $T C G$ on Nintendo DS uses Wi-Fi to Connect remote players. In this version, the trading cards are digitally represented and thus, players dô notbave the sense of the physical tangibility of the cards, which significantly decreases the pleasure and enjoyment of the game for some of players [22]. The physical angibility of the cards is essential to allow players to identify with their cards. Yu-Gi-Oh! Online also offers a similar play style on personal computers and suffers from the same law. Therefore, to overcome the described limitations of the existing systems, in Augmented TCG, we allow players to use their own collection of physical paper cards.

As described in [22], some players feel uncomfortable communicating directly with a real opponent who is a stranger. However, using only an avatar to represent the opponent, as in the existing online trading card games, does not offer enough of a sense of reality for the player. We believe that using virtual characters from animation and game stories to represent the opponent, with movements that are synchronized with those of the real opponent, would offer more of a sense of reality than the avatars used in the current online trading card games. Additionally, in Japan, trading card games are very closely associated with comic, animation and game stories, and the trading card games are a part of the stories. For this reason, players often relate to these stories and their characters while playing the games, which makes the games even more enjoyable.

In the current online trading card games, some players tend to quit the game if they are expecting to lose. This is a typical behavior of players whose only goal is winning the game. Such players feel pleasure and satisfaction only when winning the game and do not enjoy the gaming process itself. This "unfair play" is one reason why many trading card game players come to dislike playing the online versions of the trading card games [22]. Even the unfair players, however, are familiar with the comic, animation and game stories. As described before, the characters in these stories convey values and attitudes such as the importance of 
friendship and the pleasure of honest and fair play. Involving these characters in a game may remind players of these ideological messages and encourage them to behave fairly.

Many Japanese young adults admire animation and game stories and have done so since they were children. If some of the hurdles to the use of remote trading card game play are eliminated by solving the problems described in this section, then many people will continue playing the games throughout adulthood. If more young adults maintain their passion for Japanese modern culture, then a positive social change in our country could result.

\section{Enhancing Trading Card Gaming by Incorporating Virtuality}

In this section, we present Augmented TCG, a new version of a traditional trading card game that has been enhanced using virtuality. In Section 4.1, we discuss virtuality and tangibility in relation to ubiquitous computing technologies. Section 4.2 presents an overyiew of Augmented TCG and its design and implementation.

\subsection{Virtuality and Tangibility with Ubiquitous Computing Technologies}

In [13], Jordan described physio-pleasure as pleasure derived from the tangibility of a physical object. Ubiquitous computing technologies have made the physical objects that surround us more virtual by embedding computers into them. Recent advances in virtuality offer promising directions for the future. For example, proactive ambient media [20] enable us to change our attitudes and behavior or make better decisions to improve our lifestyle. In ambient media, information about a user's current situation can be represented in a peripheral display or projected onto a local artifact asing a projec Or. Virtuality is essential to proactive ambient media as it is designed to make invisible information visible.

Ambient media also demonstrate aproblem that often occurs when we introduce virtuality into our daily lives. Because the virtual objects are not material, people cannot touch or manipulate the objects in a tangible way. Thus these objects lose their sense of reality. As described in [9], future tangble technologies may solve this problem by materializing the virtual objects as if they were traditional physical objects. For example, a mobile augmented reality toolkit called Satch ${ }^{6}$ allows the development of an application that can touch and manipulate a virtua object on an mage captured by a camera. However, users still do not feel as if the object is real because they can only touch it on a touch panel. In our approach, a player can use a physical card, but the card can also be augmented by having additional information displayed on it. Additionally, the opponent is virtualized, but the movements of the virtual opponent are synchronized with those of the real opponent.

\subsection{Augmented Trading Card Game}

Augmented TCG enhances the remote trading card gaming experience in a game played by two people. The basic design approach is similar to that of the augmented reality games introduced in [27], which integrates physical and virtual items. As shown in Figure 2, the two players are located in different places. Each player's cards, in his/her duel field on the table in front of him/her, are captured by a camera and projected onto the other player's table.

The two players can communicate with each other via Skype if desired; thus, it is possible for the players to introduce themselves to each other directly instead of using virtual characters.

\footnotetext{
${ }^{6}$ http://satch.jp/en/
} 


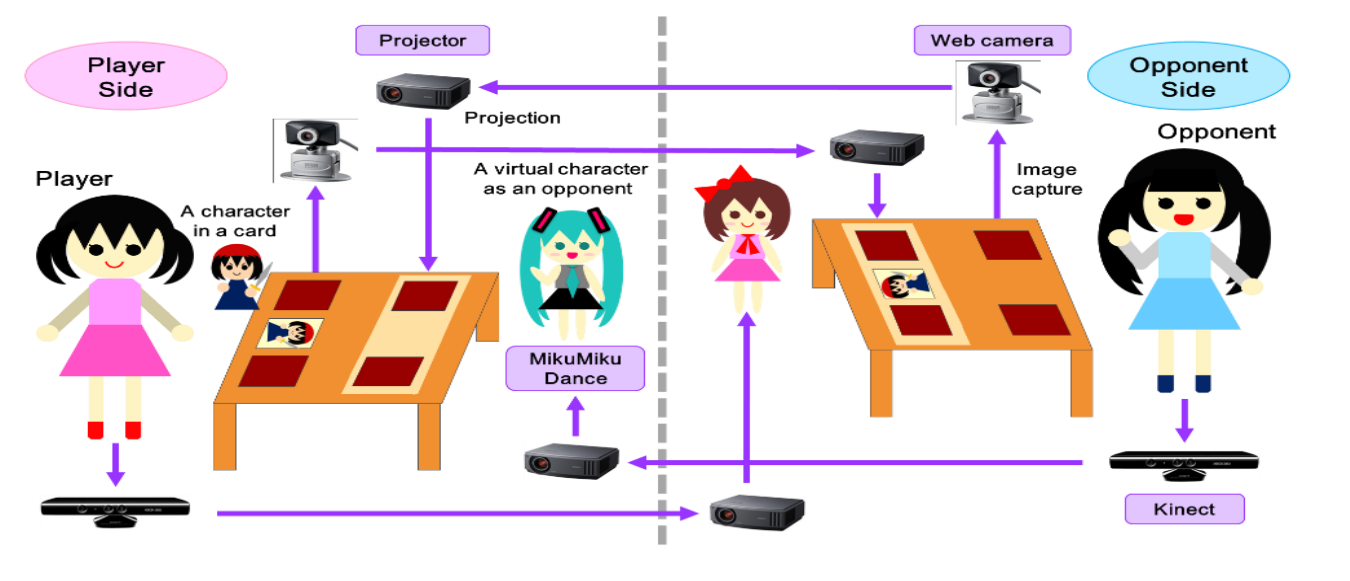

Figure 2. An Overview of Augmented Trading Card Game

Figure 3 illustrates the current game setup for a player. A camera is set up behind the small display near the player and captures the image of his/her cards. Imtages of the opponent's cards are projected onto the table using a projector that is set up/on the table. We implemented the Augmented TCG on Mirage middleware infrastructure, which is CORBAbased and hides the complex network programming from the players [26].

The following three approaches were chosen for the current version of the Augmented TCG to augment the traditional trading card game.

In the first approach, each player is represented by a 310 model of a virtual character used in popular animations and games, and 'his character is shown to the player's opponent. On a large display, shown in Figure 3, A virtual character is shown; this movements of this character are synchronized with those of the opponent. In the current implementation, MikuMikuDance ${ }^{7}$ is used to show the 3D models of the virtual characters. MikuMikuDance is free software for creating 3D movies using virtual characters. The virtual character is controlled using MS Kinect, and its mbvements are synchronized with the movements of the opponent. Plug-in software is added in MikuMikuDance to reflect captured data from Kinect into several parameters implemented in MikuMikuDance to move a virtual character's 3D model. In the current Augm nied TCG, a player can choose one of three virtual characters: Yugi or Kaiba from the Ya-Gl-Oh! animation story or Link from The Legend of Zelda ${ }^{8}$. In the $\mathrm{Yu}-\mathrm{Gi}$-Oh! animation story, Yugi is always surrounded by many friends, and his success is the result of his strong bonds with his friends who love the trading card game. Kaiba is a lonelier hero who always seeks strength in the game but does not accept help from others, even in a critical situation. In the story, however, he eventually learns the importance of friendship. Most young boys want to follow either of these two characters because of their typical, attractive and ideal personalities. We chose to also use Link in our experiments because we wanted to investigate how using a popular character from another story that is unrelated to the TCC story would affect the players' attitudes and gaming experience.

\footnotetext{
${ }^{7}$ http://www.geocities.jp/higuchuu4

${ }^{8} \mathrm{http}: / /$ zelda.com/
} 


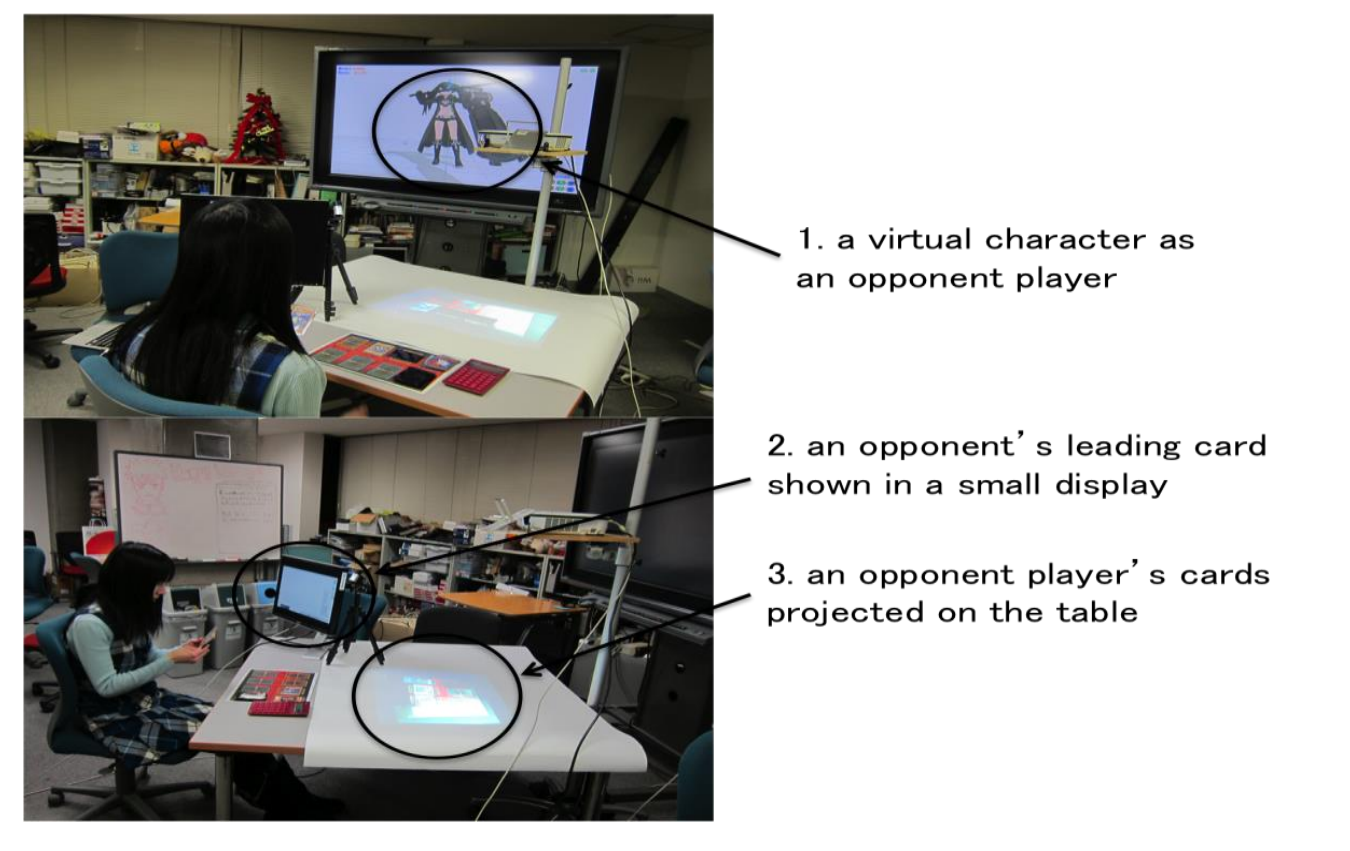

Figure 3. Augmented Trading Card Game Prototype System

In the second approach, Augmented TCG augments a player's leading card. During the game, another virtual character depictecon one of the player's cards appears on a small display near the player, as shown in Figure 3, once that card is drawn from the deck and supports and encourages the playêr hitil the end of the game.

In the third approach, the opponent's cards are shown on a small display near the player. Usually, the details of the opponent's catds are hard to see clearly and read during play. An expert player may automatically know such information by just recognizing the character on a card, but for ordinary players, this issue may decrease the pleasure of the game. In Augmented $T C G$, the quality of the projected-card images is not high enough to allow most players to gather this information; this circumstance may, however, help a player to focus on his/her own play strategy.

\section{Evaluating Augmened Trading Card Game}

We recruited of participants for our experiments. In each experimental game, the participant played against one of the paper's authors, all of whom were deeply knowledgeable about TCG and could lead and control the experiment so that all participants would play the game under the same conditions. Five participants were male, and one participant was female. All of them were 21-22 years old. The participants each had more than three years of experience with $\mathrm{Yu}-\mathrm{Gi}-\mathrm{Oh}$ ! TCG and were very familiar with the characters in the animation stories. Thus, they were well-qualified to evaluate the Augmented TCG. Before the experiments, the participants were not allowed to talk to each other and had no prior knowledge of the Augmented TCG. During the experiments, we recorded the participants' interactions with the Augmented TCG on video, and we later watched the videos to analyze each participant's play using the contextual inquiry method [2]. Finally, we conducted semistructured focus group interviews with the participants after they played. The experiments are described in detail in the following three subsections. 
For the experiments described in this paper, the rules of the game were simplified to make the duels shorter, and special predefined decks of cards were used. The decks were prepared by one of the authors, who has more than ten years of experience with $\mathrm{Yu}$-Gi-Oh! TCG and owns more than a few thousand $\mathrm{Yu}-\mathrm{Gi}$-Oh! trading cards. She is also very familiar with the TCG animation story and knows how each character structures the deck and uses the cards in the animation. For each of the virtual characters that were available to be chosen by the participants to represent them in the game, a suitable deck consistent with the animation story situation was prepared. In the current version of the Augmented TCG, the virtual character's behavior does not precisely reflect the real behavior of the player, but are exaggerated as appropriate to the current play situation.

\subsection{Virtual Character used in Animations and Games as an Opponent Player}

In two of the experiments, the participants played the Augmented TCG against a virtual character representing the opponent, as shown in Figure 3.

In the first experiment, participants could choose either Yugi or Kaba for their own character according to their preferences. After the game, we interviewed the participants about their impressions about the virtual character representing their opponent. One participant said: "I could feel I am playing against Yagi, but Yugi used inthe experiment does not offer enough." The movement of the character was sometimes vot like that of the real Yugi in the animation story. This participant also said: "I would definitely more enjoy a game against Yugi, and would like to win the game if the movement is more realistic". Another participant said: "The facial expression of the character is poor and it is a very important issue playing a game against a real person". Additionaly one participant stated: "The voice should be the same as the voice of the character in the animation story". Moreover, if the player's opponent was really ferhale, some participants found it strange because both Yugi and Kaiba are male characters.

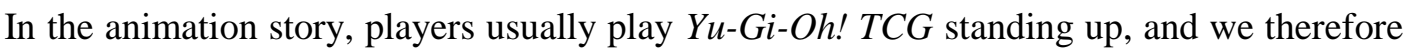
chose to have the characters standing ouring the game, but in the real world, players are usually sitting. Somesparticipants found the characters' standing positions to be unreal, but if the characters were sitting, the participants also felt that this was inconsistent with the personalities of Yugi and Kaiba.

In the second experimen, Link from The Legend of Zelda was used as the character representing the opponent. The results in this case differed depending on whether the participant liked this character. Participants who were not interested in Link did not care about the presence of Link, but if Link was their favorite character, then they found playing the game against Link more enjoyable. One of the male participants stated: "If the character is a pretty girl, I may be more excited to play the game”. Additionally, a female participant told us: "I feel that Link is my boyfriend, so playing against him increases my pleasure and positivity

\subsection{Cheering a Player by a Virtual Character in his Favorite Card}

In this experiment, a small display on the table showed the virtual character from the card that was selected in advance from the participant's deck, as shown in Figure 4. We selected Dead Master from Black $\star$ Rock Shooter ${ }^{9}$ as one of these characters because we felt that this character did not contradict or violate the atmosphere of $Y u$-Gi-Oh!. Black $\star$ Rock Shooter has two worlds. Dead Master is an enemy of Black $\star$ Rock Shooter in the other dimension world,

\footnotetext{
${ }^{9} \mathrm{http} / / /$ blackrockshooter.wikia.com/
} 
but in the ordinary world, they are very close friends. This story is a persuasive message suggesting that players need to keep and develop their friendships, even if they have serious fights with their friends.

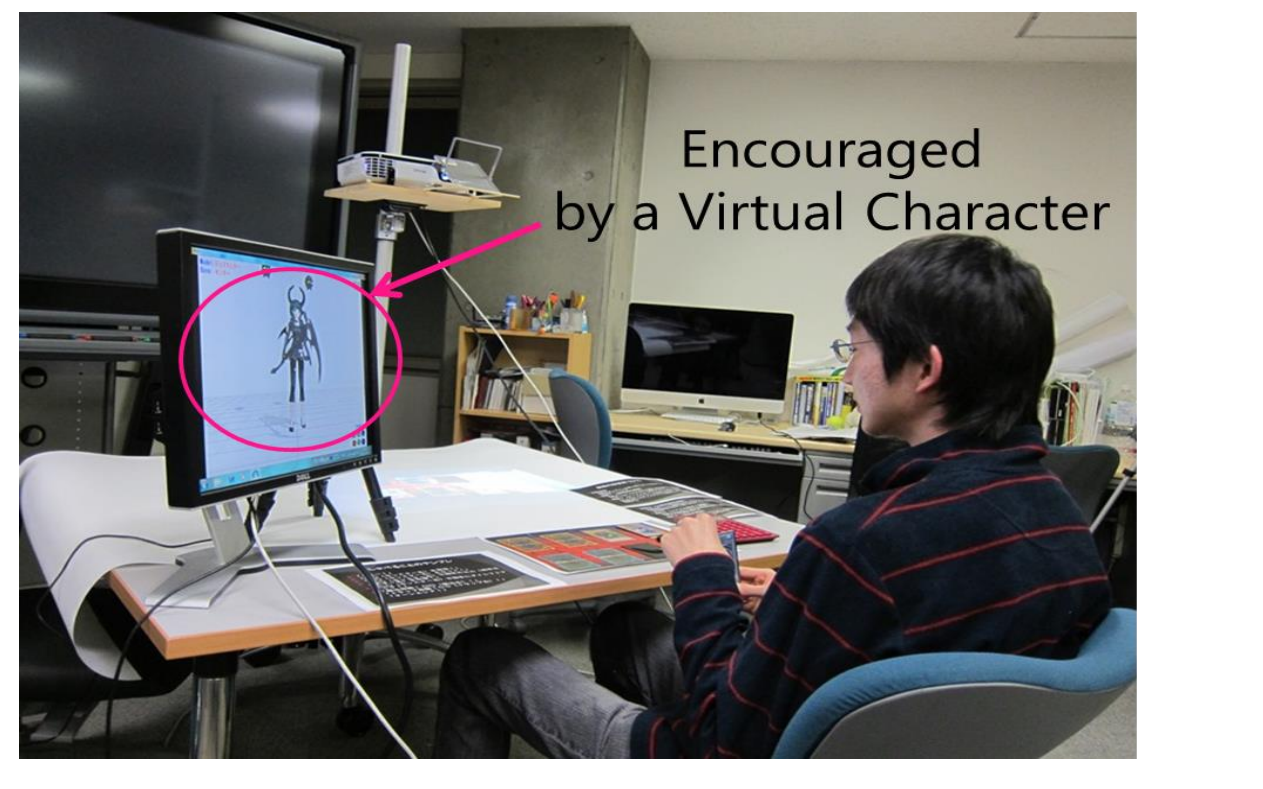

Figure 4. Encouraging a Player by a Virtual Character

As already described, in this experiment, a special deck was structured in advance for each participant depending on the chatacter he/she chose to play with. Then, during the duel, the participant always drew the card depicting Dead Master from the deck at the beginning of the game. Once that card had been drawn, a smanl display next to the player showed Dead Master until the end of the duel. Dead Master supported and encouraged the player during the game using encouraging body gestures.

After the experiment, one of the participants said: "It is desirable that the card depicting Dead Master is nol defeated by the opponent's attack.". Another player who was not interested in the character however, told us. "It is more enjoyable if the participant's favorite character encourages him". One of the other participants said: "I feel that the character does not encourage me enough aning only gestures. It is better that the character talks or advises me". This participant also told stated: "It is desirable that the character behaves like a cheerleader". Deaa Master is a serious character; therefore, players who know the animation story of Dead Master might find this type of behavior to be unrealistic due to the loss of consistency withe story. Another participant stated: "The encouragement should be like the one in the animation story". Most participants said: "The presence of the character increases the pleasure, but it is hard to consider winning the game". The participants' comments showed that they were aware that a character depicted on one of their cards appeared on the smalldisplay, although they were not informed in advance about this feature of the system.

\subsection{Showing an Opponent Player's Cards on a Small Display}

In this experiment, the card drawn by the opponent was shown on a small display near the player, as shown in Figure 5. 


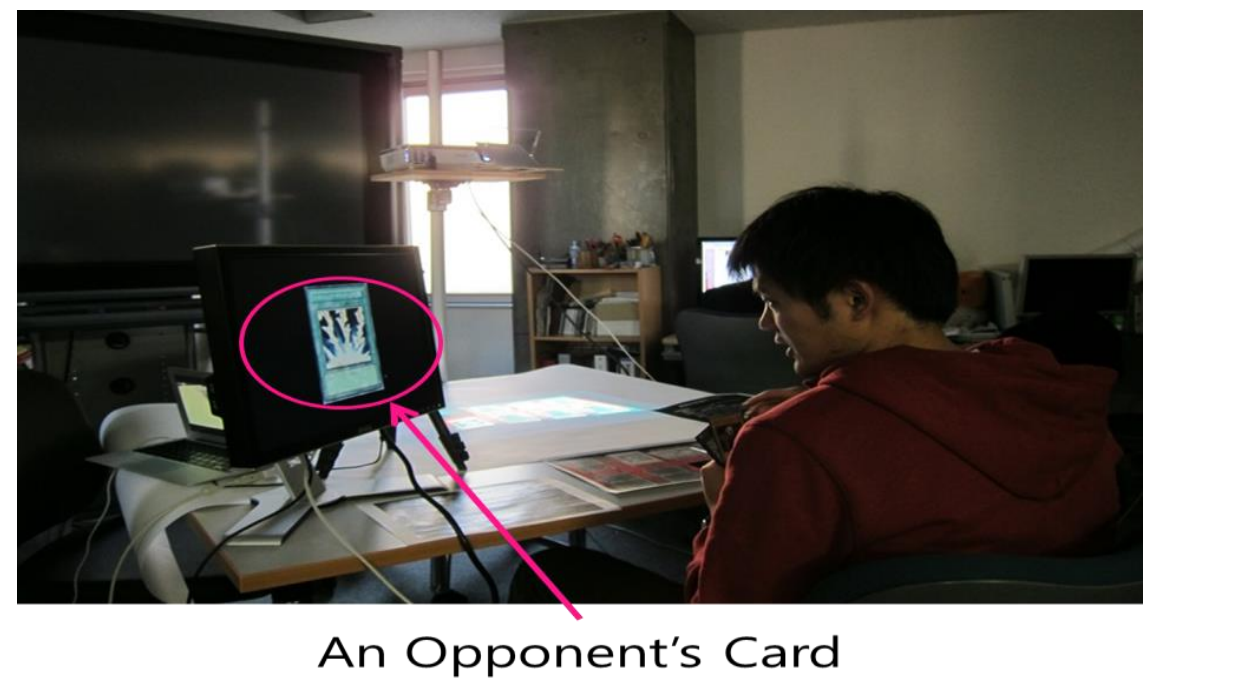

Figure 5. Showing a Card in a Small Display

As described in the scenarios presented in [22], virtual cards can significantly decrease a player's motivation and game enjoyment depending on the player's personality. If images of the opponent's cards are projected onto a player's table, it might be hard for the player to clearly see and understand the information about the characters if the words or images are small or have low resolution, making it more difficult for a player to make correct decisions. Therefore, in our set up, we showed the card draw by the opponent on a small display near the player.

The necessity of such details strongly depends on a player's goals. If a player's main goal is to win the duel, then it is important for her/him to show the superiority of his/her favorite deck, but he/she may not Gave enough 'nnowledge about the other players' cards to do so. Hence, he/she will need to see more information on the opponent's cards to play well. If a player's main goal is to play a gape and communicate with a friend, then winning a duel will be relatively unimportant. In such a case, the information on the displayed card is not very important be ause he/she car anvays ask his/her friend (the opponent) for more information if necessary, and such communication could even strengthen the friendship. If a player's goal is to collect cards, then he/she will be familiar with most of the cards, and just seeing the shape of the illustration onthe card will be enough for him/her to know its functionality. Thus, for $\mathrm{him} / \mathrm{her}$, it is not important to see the small details of the cards. However, we believe that being able to show detailed card information will always have a positive impact on the enjoyment of the game.

\subsection{The Effects of Using a Virtual Character from a Popular Animation Story}

The most important characteristic of Augmented TCG is the use of a virtual character to augment traditional TCG. In this section, we discuss our observations, from our experiences with designing and playing Augmented TCG, about the effects of using a virtual character in this way.

As already mentioned, most of the participants in the experiments, all of whom had watched the $\mathrm{Yu}$-Gi-Oh! animation story, felt that including popular virtual characters from the animation in the game can make it more enjoyable and exciting. The desire to imitate these characters may also become an incentive for the users to change their behavior. Negative 
feedback may be used to achieve moral play, but changing a user's general attitude is not easy [19]. The most important insight is that it is possible to use a virtual character as a symbol that reminds the player of the character's story during the game, and in this way, the story may convey values such as the importance of friendship, honesty, and thoughtfulness. This approach could have the power to change the player's attitude towards "fair play".

As mentioned above, the results of our experiments and interviews with participants have indicated that the relative realness of the virtual characters, such as their facial expressions, movements and behavior, is important for players' enjoyment of the game. Realness is an important criterion for evaluating a design [23, 24]. In Augmented TCG, the virtual character's behavior should be consistent with the character's behavior in the animation story, and this consistency is important to players' sense of their realness in the game. For example, cards that are not used by the character in the animation should not be used in the game, and the movements of the virtual character should be consistent with its movements in the animation. The number of cards owned by the character in the game should be consistent with the corresponding number in the animation as well. Excessively realistic expressions, however, may lead to the uncanny valley problem [16] if the achieved realness is perceived as not being completely realistic. The balance between the realness and the virtuality of the virtual characters is important for design strategies when introducing virtaliality into the real world. In the second experiment, the participants were not very familiar with the chosen virtual character, but in some cases, it caused a sense of incongruty during the game if they were aware that the character was from another animation story anrelated to $\mathrm{Yu}$-Gi-Oh! TCG.

Most people, especially Japanese people, like fictional stories in which the characters are able to do whatever they want by using hidden magic apitities. For example, many Japanese people attach pictures of favorite characters to their bags and mobile phones. These actions increase their identification with then bags and mobile phones. If the stories become more pervasive in our daily lives, then the boundary between the real world and the fictional world becomes more blurred. Therefore, fictional stories can be used to enhance our daily activities. These stories can increase people's positive thinking in the real world and enhance their selfefficacy to do what they want because, through participation in these games, people can develop the illusion of haying special extraordinary abilities. In their daily lives, people may not feel as if they are able to change heir undesirable attitudes and behaviors because as daily life becomes more and more complicated, we do not have enough time to consider the importance of the more desirable behaviors.

Many Japanese animation and game stories emphasize the importance of positivity. Thus, the characters in these stories can help to increase people's positivity as they play a game. This is especially true for many young Japanese people who have grown up with fictional stories, such as game, animation and science fiction stories. Positivity is an important factor that can increase people's self-efficacy to solve difficult problems, such as those involving environmental sustainability or human well-being, which has been observed in psychology research [8]. Positivity is very useful for making our lives more meaningful [18] and is essential for success in our life. Our approach to incorporating virtuality into the real world is a possibly powerful way to increase people's positivity.

\section{Introducing Virtuality into Transmedia Storytelling}

Enhancing real world games, such as TCGs, with fictional stories is a promising direction for designing a new form of transmedia storytelling [5, 25]. In this section, we discuss some design implications of incorporating virtuality into physical artifacts that may be important for designing future daily artifacts enhancing with transmedia storytelling. In transmedia 
storytelling, a fictional story is told across multiple media platforms, which are distributed throughout the real world. In our approach, we use some items from a story to remind people of the story. For example, in the Augmented TCG, a trading card is an item that appears in the story, and Yugi and Kaiba as virtual characters also remind the players of the story. Virtual characters are designed to be remembered for a long time; therefore, a character from an animation story is suitable to be used as a reminder of the story.

Seichi Junrei is a typical geek culture activity in Japan and is related to Japanese animation, manga (comic) and games. "Seichi" means "Holy Land", "Junrei" means "Pilgrimage". In Seichi Junrei, people visit famous locations from animation, manga and games. Anime fans take pictures at a specific location at the same screen/angle as it appears in the animation and then upload the pictures to their blogs. The most important aspect of Seichi Junrei is that something is brought from the fictional story to the real world. The fans create new stories using these pictures and the virtual characters from the fictional stories, and share them within their community.

Recent animated movies use many scenes from the real world to increase the realness of the fictional movies. A user of Seichi Junrei tries to find the scene that appears in the movie in the real world and takes a photo of the scene. The user enjoys finding these scenes and visiting the locations of the scenes. Then, he/she takes a photo, as in a scavenger hunt. The photos are usually uploaded to the Web, and many other people enjoy looking at the photos.

In Japan, especially, animated movies have recently been used to promote the local districts that appear in the stories. Some districts work to attract people who like the stories by offering extra new original stories to thosewho isit the districts. This strategy suggests that it is possible to promote districts using fietional stories. If real products that the district is known for appear in the stories, then if W11 incresse the leeling that the stories are realistic, especially if the audience is familiagwith the products. If the stories are attractive, they will also promote the products to people who do notive in the district.

The above phenomenon, which is an interesting example of harmonizing the real world with the fictional world, suggests new possibilities for incorporating virtuality into the real world. Stories being embodied in the real world through transmedia storytelling increases our sense of their realness. By incorporating virtual characters from the stories into the real world, each person can create bis/her own story based on these stories, as in Seichi Junrei.

As already described, virtual characters from animations and game stories are widely used in multiple media channels. Por example, in Pokémon ${ }^{10}$, synergy among games, movies, and television programs is used to make the Pokémon story more popular and to make the story pervasive in its fans' daily lives. Additionally, in the $Y u$-Gi-Oh! animation story, the story teaches its audience how to play the TCG game and why the game is attractive. Using multiple channels to communicate messages to people through transmedia storytelling is an effective way to convey the messages because each channel can convey the message in a special way. This approach is also typically used in current advertising because one medium alone annot deliver advertising to a large audience of people. One of the problems in using virual characters is copyright. There are many free 3D models for MikuMikuDance on the Web, but many have been deleted due to copyright issues. The freely available models offer new possibilities to enhance games because the models can be easily customized. In Japan, it is popular to create new characters and stories from existing ones. Using a customizable virtual character in Augmented TCG may create a new playing style of TCG, and the new stories created about the characters may enhance the story's message.

\footnotetext{
${ }^{10}$ Pokémon is a media franchise published and owned by Japanese company named the Pokémon Company and its original videogame created by Satoshi Tajiri in 1996.
} 


\section{Conclusion and Future Direction}

This paper presents the design and some observations on the usage of an Augmented TCG that enhances remote trading card game play by introducing virtuality. Specifically, we have discussed the impact of introducing virtual characters used in animation and game stories into the game. We have reported on experiments with Augmented TCG and presented the results and their design implications.

In the current Augmented TCG, a player must manually teach the system which cards he/she likes to use. If the system could automatically detect the player's cards, then the usability of the system would be dramatically improved. The automatic detection of cards can be easily realized by inserting an RFID into each card, similar to the approach described in [6]. In our current system, it is sometimes difficult to clearly see the opponent's cards, and in such case, a player must explain which card he/she is using and the effect of the card. If detailed information about each card were automatically shown on the duel field, then the players would not need to engage in such extra communication using a voice connunication system or a chat system. Additionally, in Augmented TCG, it wôld be easy to detect mistakes made by players, which would be very helpful because sometimes it is hard even for a semiexpert player to understand the complex rules of a game.

As described in Section 3, the current version of Augmented TCG uses physical cards. To augment the cards, we displayed special effects on then. This approach increases a player's cognitive load because he/she needs to look at both his/her eards and at the display. When participants became enthusiastic about the game, they usually forgot about the existence of the display. It would be better to use more immersive technologies to enhance the physical cards directly. We suggest two possible appoaches for solving this problem. The first would be to use a projector to project the special effects onto the cards. It is, however, not easy to install a projector to accurately project the special effects onto a player's cards. The second approach would be to use a head mounted display. This would require accurate identification of the movements of a player's head. In the $\mathrm{Yu}-\mathrm{Gi}$-Oh! animation story, a player usually wears a head mount display so this approach may be accepted as natural by people who are familiar with the animation story.

Realness is a very Important criterion for the design and augmentation of physical artifacts. This does not mean that we need to offer the same physical items that we have used before. Adding virtuality offers the possibility of offering more sophisticated user experiences. It is important to consider how a player feels about physical items, and this consideration remains important after virtuality is introduced. The value based design framework described in [21] will be an essential tool. For example, the facial expressions of ap opponent offers useful information for formulating a game strategy, and these facial expressions becomes the informative value defined in the framework. However it is not necessary to present the opponent's exact facial expressions; only the information from the expressions that the player needs for playing the game is needed. The info mation may, for example, be offered in the form of textual messages. Additionally, as described in Section 3, the tangibility of cards is important. The value that players place on the cards is their economic value. If they feel that the cards are worth collecting, then the cards are satisfying a need. If people see virtual cards as having the economic value, then they may not feel that the cards need to be tangible. Gaming will be an important medium in the future because the interactivity of gaming gives players a feeling of autonomy. As described in [4], autonomy is one of the most important factors in a healthier lifestyle. As described in this paper, the positivity of typical Japanese animation stories can help to increase our self-efficacy. Self-efficacy is 
key for increasing our quality of life. The insights described in this paper should be considered in future projects using virtuality to enhance physical artifacts. In the future, the approach will allow us to design new artifacts that will help to increase people's self-efficacy and positive thinking and to enhance their intrinsic motivation to tackle serious social problems.

\section{References}

[1] J. Baudrillard, The Consumer Society: Myths and Structures, Sage Publications Ltd, (1998).

[2] H. Beyer and K. Holtzblatt, "Contextual Design", Morgan Kaufmann, (1999).

[3] S. Cuendet, E. Bumbacher and P. Dillenbourg, "Tangible vs. Virtual Representations: when Tangibles Benefit the Training of Spatial Skills", Proceedings of NordiCHI 2012, (2012).

[4] E. L. Deci and R. M. Ryan, ““"What” and "Why” of Goal Pursuits: Human Needs and the Self-Determination of Behavior”, Psychological Inquiry, vol. 11, (2000), pp. 227-268.

[5] T. Dowd, M. Fry, M. Niederman and J. Steiff, "Storytelling Across Worlds", Transmedia for Creatives and Producers, Focus Press, (2013).

[6] W. Hazenberg and M. Huisman, "Meta Products: Buidling the Internet of Things, BIS Publisher, (2011).

[7] C. Floerkemeier and F. Mattern, "Smart Playing Cards - Enhancing the Gaming Experience with RFID", Proceeding of the 3rd Int. Workshop on Pervasive Gaming Applications, (2006), pp. 79-88.

[8] B. L. Fredrikson, "Positivity: Top-Notch Research Reveals the 3 to 1 Ratio That Will Change Your Life", Three Rivers Press, (2009).

[9] H. Ishii and B. Ullmer, "Tangible bits: towards seamless interfaces between people, bits and atoms", Proceedings of the SIGCHI Conference on Human Factors in Computing Systems, (1997), pp. 234-241.

[10] T. Iwata, T. Yamabe and T. Nakajima, "Augmented Reality Go: Extending Traditional Game Play with Interactive Self-Learning Support", Proceedings of the 17th IEEEE Conference on Embedded and Real-Tie Computing Systems ad Applications, (2011), pp $105-114$.

[11] K. Fujinami and T. Nakajima, "Sentient artefacts: Acquiring) user's context through daily objects", Proceedings of the 2005 international conference on Embedded and Ubiquitous Computing, (2005), pp. 335344.

[12] K. Fujinami, F. Kawsar and T. Nakallma, "AyareMirror: A personalized display using a mirror", Proceedings of the $3^{\text {rd }}$ International Conference on Pervasive Computing, (2005), pp. 137-150.

[13] P. W. Jordan, "Designing pleasurable products. An introduction to the new human factors", Routledge, (2002).

[14] F. Kawsar, K. Fujinami and T.. Kakajima "Augnenting everyday life with sentient artefacts", Proceedings of the 2005 joint conference on Smart objects and ambient intelligence: innovative context-aware services: usages and technologies, (2005), pp 141-146.

[15] C. Magerkurth, A. David Cheok, R.L. Mandryk and T. Nilsen, "Pervasive Games: Bringing Computer Entertainnent Back to the Rear World", ACM Computer in Entertainment, vol. 3, no. 3, (2005).

[16] M. Mori, 'On the Uncanny Valley", Proceedings of the Humanoids-2005 Workshop, (2005).

[17] S. Marzano and E. Aarts, "The New Everyday View on Ambient Intelligence", 010 Publisher, Rotterdam, (2003).

[18] J. McGonigal, "Engagement Economy: the future of massively scaled collaboration and participation", Institute for the Future, | SR-1183, (2008) September.

[19] T. Nakajima andV. Lehdonvirta, "Designing Motivation in Persuasive Ambient Mirrors", Personal and Ubiquitous Computing, vol. 17, no. 1, (2013), pp. 107-126.

[20] T. Nakajima, T. Yamabe and M. Sakamoto, "Proactive Ambient Social Media for Supporting Human Decision Making", Proceedings of the $8^{\text {th }}$ International Conference on Ubiquitous Intelligence and Computing, (2011), pp. 25-39.

[21] M Sakamoto, T. Nakajima and T. Alexandrova, "Digital-Physical Hybrid Design: Harmonizing the Real World and the Virtual World", Proceedings of the 7th International Conference on the Design \& Semantics of Form \& Movement, (2012), pp. 211-222.

[22] M. Sakamoto, T. Alexandrova and T. Nakajima, "Analyzing the Effects of Virtualizing and Augmenting Trading Card Game based on the Player's Personality”, Proceedings of The Sixth International Conference on Advances in Computer-Human Interactions, (2013).

[23] M. Sakamoto, T. Nakajima and S. Akioka, "Designing Enhanced Daily Artifacts based on the Analysis of Product Promotions using Fictional Animation Stories", Proceedings of International Conference on Active Media Technology, (2013).

[24] M. Sakamoto and T. Nakajima, "An Analysis of Composing Multiple Fictional Stories and Its Future Possibility”, Proceedings of $15^{\text {th }}$ International Conference on Human Computer Interaction, (2013). 
[25] M. Sakamoto, T. Alexandrova and T. Nakajima, "Augmenting Remote Trading Card Play with Virtual Characters used in Animation and Game Stories - Towards Persuasive and Ambient Transmedia Storytelling", Proceedings of the 6th International Conference on Advances in Computer-Human Interactions, (2013).

[26] E. Tokunaga, A. van der Zee, M. Kurahashi, M. Nemoto and T. Nakajima, "A Middleware Infrastracture for Building Mixed Reality Applications in Ubiquitous Computing Environments", Proceedings of the 1st Annual International Conference on Mobile and Ubiquitous Systems, Networking and Services, (2004).

[27] T. Yamabe and T. Nakajima, "Playful Training with Augmented Reality Games: Case Studies towards Reality-Oriented System Design”, Multimedia Tools and Applications, vol. 62, no. 1, (2013), pp. 259-286.

\section{Authors}

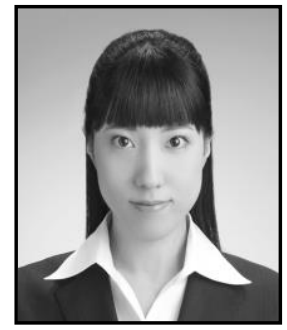

Mizuki Sakamoto is a Ph.D. candidate in the Department of Computer Science and Engineering at Waseda University Her research interests are in game studies, game design, virtual economy and ubiquitous computing.

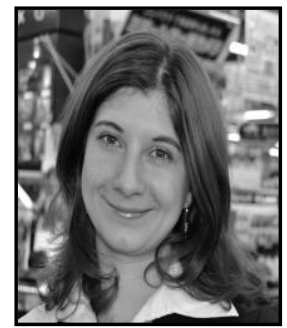

Todorka Alexandrova received herB.Sc. and M.Sc. degrees in mathematics from Sofja University, Bulgaria in 2000 and 2003, respectively. She received her D.Eng. degree from the University of Electro-Communications, Tokyo. Lapan in 2008. Currently she is an Associate Professor at the Anternational Center for Science and Engineering Pograms, Waseda University, Tokyo, Japan. Her current research interests are in information security, cryptography, crowdsourcing, gamification and ubiquitous computing.

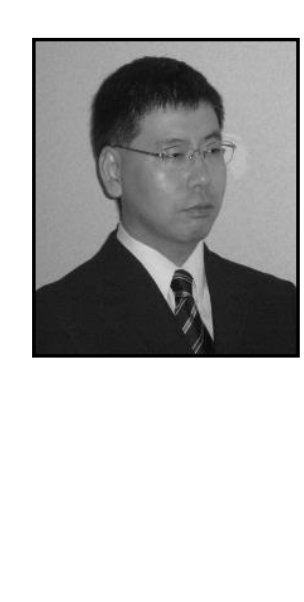

Tatsuo Nakajima is a professor of Department of Computer Science and Engineering in Waseda University. He received Dr. Sci. in Electronic Engineering from Keio Univ, in 1990. He worked in Carnegie Mellon University from 1990 to 1992, and worked on the development of the Real-Time Mach microkernel. In 1993, he moved to Japan Advanced Institute of Science and Technology until 1999. He worked on adaptive multimedia and mobile systems top on the microkernel. In 1998, he was a research engineer of Olivetti and Oracle Research Lab., and worked on the implementation of the CORBA system. From 2000, he moved to Waseda University and worked on ubiquitous computing and operating systems for embedded systems. His group has developed middleware for home appliances, AR-based user interface, persuasive services and smart objects in the Ubicomp research. His group also developed a virtualization layer for multi-core processor based embedded systems. In 2005, he has a visiting research fellow of Nokia Research Center, Helsinki and a visiting professor of University of Helsinki. 
International Journal of Smart Home

Vol.8, No.2 (2014)

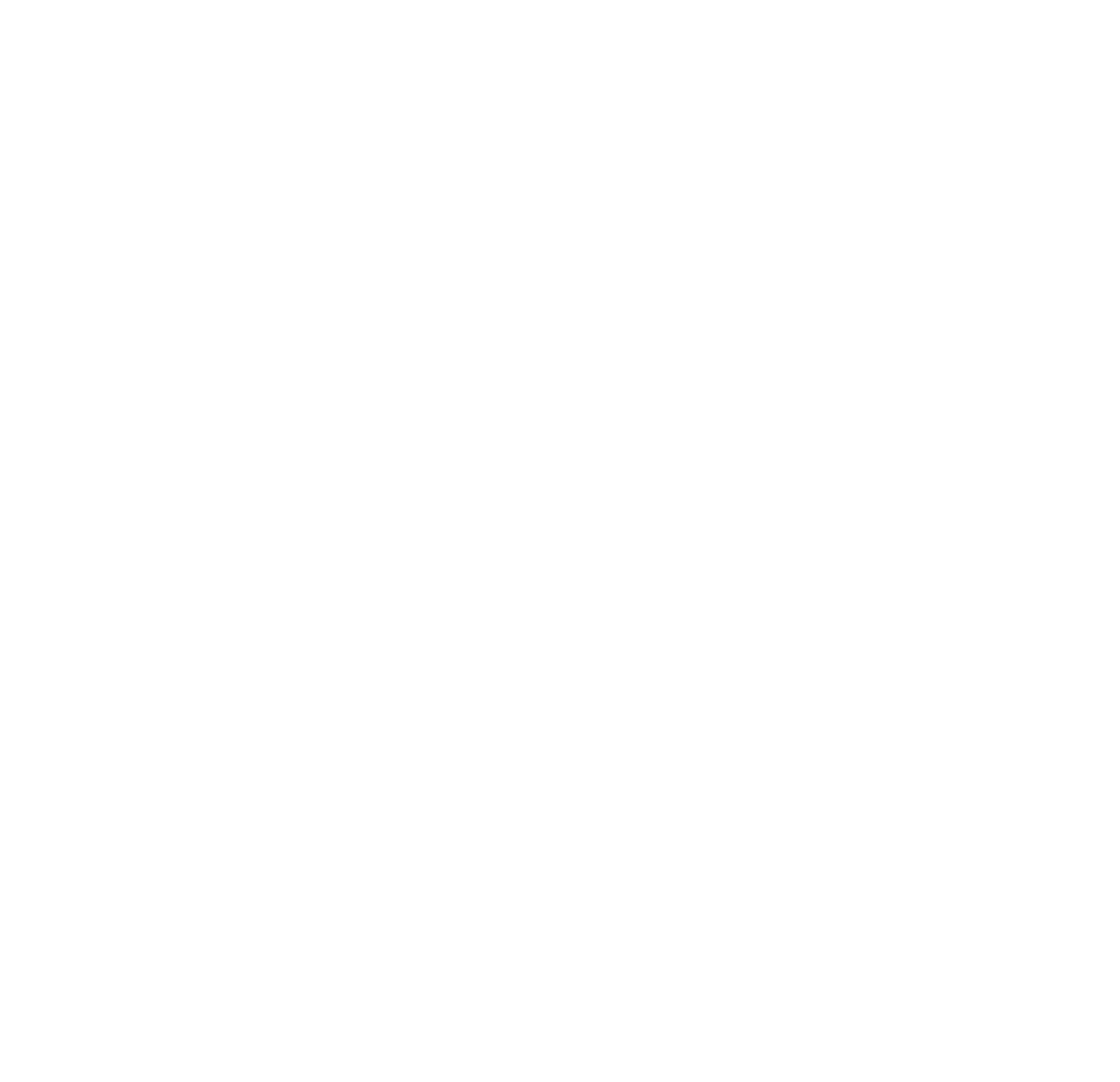

